\title{
Auf dem Weg zu einem ökozentrischen Management
}

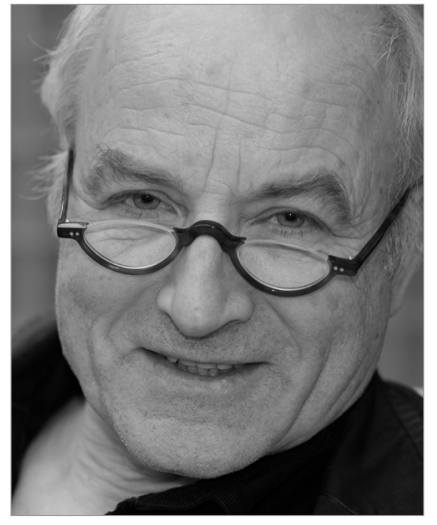

VON ANDREAS STRUNK Prof. Dr. Andreas Strunk lehrte bis zu seiner Pensionierung an der Hochschule Esslingen das Fach "Organisationswissen für die Soziale Arbeit". Er ist Mitbegründer der Zeitschrift SOZIALwirtschaft und des Informationsdienstes SOZIALwirtschaft aktuell. prof.strunk@t-online.de

\author{
Organisationen werden oft als Maschinen \\ angesehen, die man nur richtig warten und bedienen \\ muss. Diese Eindimensionalität hat etwas mit \\ Planungsoptimismus zu tun, mit der in der Regel nicht \\ hinterfragten Wirksamkeit von Management und \\ dem diesem hinterlegten Plangungsverständnis.
}

Der Autor des vorliegenden Textes befasst sich seit Anfang der Neunzigerjahre des vergangenen Jahrhunderts mit Fragen des Sozialmanagements. Seit 1991 füllt der Autor einen Zettelkasten und beobachtet, in welchen Zusammenhängen der Begriff (und damit die Praxis) »Management « auftauchen. Bisher gibt es im Zettelkasten 47 Hinweise. Von A (Affekt Management) bis Z (Zeitmanagement).

Der Inhalt des Zettelkastens ist sicher - statistisch betrachtet - nicht repräsentativ. Er hängt unter anderem vom Leseverhalten und der Netzwerkeinflechtung des Autors zusammen. Er lässt aber eine Reihe von Aussagen in qualitativer Hinsicht zu.

Zunächst ist auffallend, dass fast der gesamte Lebensraum und die damit zusammenhängenden Sachverhalte zum Gegenstand von Management gemacht werden (Körper, Umwelt, Natur, Tiere, Mobilität, Fahrzeuge, Räume etc.). So verweisen Artikel, die im Kontext von »Genmanagement « stehen, auf biotechnologische Visionen, in denen deutlich werden soll, dass der »homo sapiens" durch einen evolutionären Sprung abgelöst wird durch einen »homo deus «. Treiber sollen sein: Bio Engeneering, die Kreation von Cyborgs und die Entwicklung anorganischen Lebens. (1)

Dahinter steckt eine Fortschrittsgläubigkeit, die erschrecken kann, weil wir zum Beispiel an der Entwicklung des
Internets erlebt haben, dass sich diese technologische Innovation ohne die Beteiligung demokratischer Kontrollen entlang der Implementation ereignet hat. Demokratische Kontrollen entwickeln sich erst im Nachhinein.

In Anlehnung an die Argumentation des Soziologen Arnold Gehlen (2) spricht der Politologe und Philosoph Hans-Peter Hempel von »Machbarkeitswahn und Daseinsgefräßigkeit im Biotechnischen Zeitalter« (Berlin/Wien 2003). Hempel spricht von einem verhängnisvollen Viereck zwischen

- kapitalistischen Verwertungsinteressen, - an diese gebundene Wissenschaft- und Technikagenturen,

- einer dressierten Konsumentennachfrage

- und einem kompensierenden Wohlfahrtsstaat.

Als ich Hempels Buch gelesen hatte, kam mir die neutestamentliche Johannesapokalypse in den Sinn. Erinnern wir uns, der Autor dieser Dystopie malt ein Bild, das sich wie folgt zusammenfassen lässt: Da gibt es eine Stadt (»Babylon«), in der die Menschen und ihre »Großkaufleute « sich vom »Hurenwutwein « haben betrunken machen lassen. Ihnen droht das Gottesgericht. Ihr Verhaltensmodell entspricht nicht einem gottwohlgefälligen Leben. (3) 


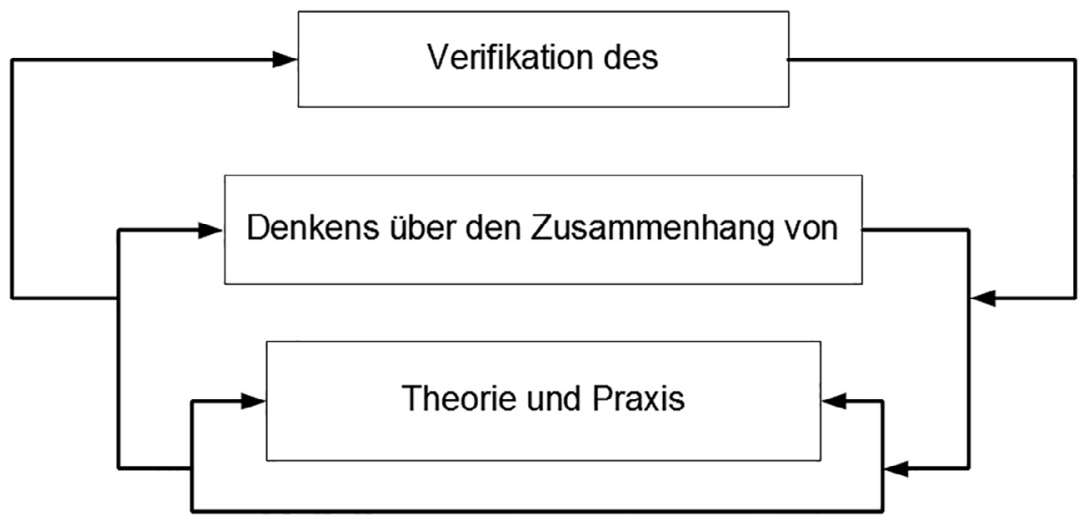

Theorie-Praxis-Relationen müssen eingebettet sein in ein Nachdenken über ihr Umfeld und über mögliche Bewertungen.

Die Theologin Ulrike Sals interpretiert dieses Weltuntergangsszenario wie folgt: "So sind hier die Kaufleute diejenigen, die sich prostituieren, und zwar an einer Stadt-Frau, die aber in den Augen Gottes eine Prostituierte ist. Die Stadt-Frau aber hat ökonomische Potenz, und zwar totale: das wirtschaftliche System ist ein Monopson, ein Nachfragemonopol. Es ist ein im doppelten Sinn perverses System, dass Johannes mithilfe der Engel hier durchschaut.« (4)

Die Handelnden folgen einer Ideologie und einem entsprechenden Handlungsmodell, in dem sie sich wechselseitig verkaufen. Es geht also um einen mehrfachen Verstrickungszusammenhang. Auf der einen Seite geht es eher um die Missachtung einer Würde des Leibes, auf der anderen Seite eher um die eindimensionale Fokussierung auf finanziellen Ertrag und auf beiden Seiten auch um die Verweigerung einer Auseinandersetzung mit einer spirituellen Grundlage unseres Lebens in der Welt.

»Wir leben in einer Zeit, die randvoll mit Problemen ist. Und umstellt von Problemen, leben wir mit der Frage, wie wir, ja ob wir mit ihnen fertig werden können. Bürger und Öffentlichkeit nehmen dadurch teil an den ins Gigantische gewachsenen gesellschaftlichen Anstrengungen. Denn die moderne Gesellschaft ist ja vor allem zu einer ungeheuren Veranstaltung zur Lösung ihrer Probleme geworden ...«

Das sind einleitende Sätze aus dem Kapitel »Die Glaubensgeschichte der Moderne« (S. 126), die der Soziologe Friedrich H. Tenbruck formuliert hat. (5)

In dieser Glaubensgeschichte weist Tenbruck darauf hin, dass in den vergangenen dreihundert Jahren seit der
Aufklärung immer wieder der Versuch unternommen wurde, die Rolle der Religion zu ersetzen. Dies sei jeweils ein Prozess gewesen von Suche, Hoffnung und Enttäuschung. Bis schlussendlich unsere Glaubensgeschichte »verhüllt« und »unkenntlich « geworden sei. Offen sei, ob eine "von einem neuen Glauben getragene Kultur« sich einstellt (a. a. O., S. 142), denn Wissenschaft alleine sei nicht in der Lage, die Fragen zu beantworten, was die für die Menschen als legitim anzuerkennende Ordnung sei. Die Sinnfrage zum menschlichen Leben blieben mithin im reinen Wissenschaftskontext unbeantwortet.
Auch im Kontext von Politik findet man offensichtlich kaum eine Antwort. So scheint Gregor Gysi mit einer »negativen Theologie « - auf diese Orientierung gehe ich später ein - zu sympathisieren, wenn er, befragt, was gegen die Konsequenzen von Neoliberalismus und Rechtsruck zu unternehmen sei, wie folgt antwortet: »Ich glaube zwar nicht an den da oben, aber ich fürchte eine gottlose Gesellschaft.«(6) Er geht davon aus, dass ein für die Gesellschaft notwendiges »Wertegerüst« von den Kirchen aufgestellt werden könnte. Der Kapitalismus könne das nicht, der Sozialismus ebenso wenig.
Was Gysi hier argumentiert, könnte auf eine Aufhellung unserer Glaubensgeschichte hinauslaufen.

\section{Was das alles mit Management zu tun hat}

Offensichtlich ist es so, dass der ubiquitären Gebrauch von Management und die entsprechende Praxis in nahezu allen Sektoren unseres Lebensraumes auf dem Konzept einer prinzipiellen Lösbarkeit der im Lebensraum herrschenden Problemen basiert. Die Welt wird als eine triviale Maschine modelliert.

Diese Eindimensionalität hat etwas mit Planungsoptimismus zu tun, mit der in der Regel nicht hinterfragten Wirksamkeit von Management und dem diesem hinterlegten Plangungsverständnis.

Dazu hat Arnold Gehlen eine bemerkenswerte Anmerkung formuliert: »Dieser Planungsoptimismus, als die moderne Form des Vernunftglaubens, hat wie dieser etwas Instinktives, Emotionales und Unkontrolliertes, der Glaube an die innere Wahrheit von Denkmodellen und der an die Beherrschbarkeit auch der größten, kompliziertesten Verhältnisse durch sie, wie sie in ganzen Gesellschaften vorliegen, ist selbst rational nicht begründbar. Er hat allerdings auch, wie jeder echte Glaube, kein Bedürfnis nach einer Begründung.«(a. a. O., S. 77)

\section{"Die moderne Gesellschaft ist zu einer ungeheuren Veranstaltung zur Lösung ihrer Probleme geworden"}

Gehlen beklagt zudem eine verhängnisvolle Zirkularität, einen Teufelskreislauf: Das gegenwärtige gesellschaftliche Gefüge sei einerseits getrieben von einem »Recht auf Wohlleben « und andererseits würden durch die Produktion und $\mathrm{Au}$ tomatisierung von Konsumbedürfnissen die notwendigen gesellschaftlichen Grundlagen und Ressourcen in wachsendem Maße zerstört. Der Autor ruft in dem Kapitel »Umsturz und Askese (a. a. O., S. 75-81) zu Enthaltsamkeit und Selbstbeherrschung auf. Er spricht von einem notwendigen Asketismus .... »eine Stärkung des inneraffektiven Zusammenhanges, ein Mehr an Integration und 
Fassung der Person, verbunden mit einer Verschärfung der sozialen Antriebe, eine Steigerung der geistigen Wachheit - also ein Sichäußern gerade durch Sichkonzentrieren« (a. a. O., S. 79).

Das, was mit einem »Mehr an Integration und Fassung der Person « gemeint ist, kann in einer Psychologie der Persönlichkeit auch als Spiritualität gekennzeichnet werden. (7) Das hängt wohl damit zusammen, dass wir uns in einer Phase der
- Erfahrung, dass das Leben ein »Problem « darstellt

- die »Problemlösung « bedarf einer integralen Kompetenz (Vernunft, Liebe, Mitgefühl, Mut)

- der Mensch darf nie Mittel zum Zweck werden

- Loslassen des eigenen »Ich"von der eigenen Gier

- Erfahrungsbereitschaft gegenüber Transzendenz.

\section{"In fast allen spirituellen Schulen gibt es eine Triade: Innehalten, Umwenden, Loslassen"}

"zweiten Aufklärung « befinden, in der erstens deutlich wird, dass es neben der Entwicklung instrumenteller, kognitiver und emotionaler Intelligenzformen auch um die Entwicklung einer spirituellen Intelligenz geht und dass zweitens Politikentwicklung ohne die Beachtung religiöser Grundbedürfnisse sinnlos erscheint. (8)

Welche Konsequenzen hätte die Ausweitung des Intelligenz-Verständnisses auf die Fragestellung, die Gegenstand des vorliegenden Textes ist?

Zunächst geht es um die Anerkennung von Mehrdimensionalität, wenn es um Management und Planung unseres gesamten Lebensraumes geht.

Es reicht nicht aus, Gestaltungsfragen im Sinne einfacher Theorie-Praxis-Relationen zu reflektieren. Wir brauchen offensichtlich weitere Perspektiven: das Nachdenken über den Sinn von TheoriePraxis-Relationen und eine Perspektive, mit der wir die Werte festlegen, um über den Sinn des Nachdenkens über Theorie-Praxis-Relationen urteilen $\mathrm{zu}$ können.

Wenn wir uns um diese weiteren Perspektiven bemühen, dann bemühen wir uns um einen Vorgang, den man als Verifikation bezeichnen kann (vgl. Grafik).

Es geht dann um Analysen, die auf sogenannte X-Erfahrung basieren, wie das der Psychoanalytiker Erich Fromm ausdrückt. Dieses Erfahrungsfeld beschreibt der Autor als »theistisch ebenso wie einer nicht-theistischen religiösen Erfahrung« zugeordnet. (9) Gefordert sind die Entwicklung und Übung von spiritueller Intelligenz. Fromm benennt folgende Aspekte von X- Erfahrung
In diesem Zusammenhang spricht Fromm von einer "negativen Theologie« und verweist auf eine Aussage des Mystikers Meister Eckart (10): »Ich überlegte mir einst (es ist noch nicht lange her), dass ich ein Mensch bin, das ist auch einem andern Menschen mit mir gemein; dass ich sehe und höre und esse und trinke, das tut auch ein anderes Tier; aber dass ich bin, das ist keines Menschen sonst als allein mein, weder eines Menschen noch eines Engels noch Gottes, außer sofern ich eins mit ihm bin. Alles, was Gott wirkt, das wirkt er in dem Einen sich selbst gleich, und doch ist es in den Werken einander gar ungleich."

Nun adressiert Fromm seine Aussagen an Individuen, wie sich das für einen Psychoanalytiker gehört. Die Frage ist jetzt, wie integrale Kompetenz als Treiber für Organisationsentwicklung wirksam werden kann. Es ginge also um den Zusammenhang von individuellem und organisationalem Lernen.

In fast allen spirituellen Schulen - ob sie nun theistisch oder nicht-theistisch orientiert sind - gibt es die Triade »Innehalten, Umwenden und Loslassen «. Die Beachtung der Triade kann sehr praktische Konsequenzen haben. Dazu ein Beispiel aus der Wohnungslosenhilfe: Wir haben über die damalige vorherrschende Praxis der Hospitalisierung von Wohnungslosen in Asylen nachgedacht (Innehalten) und sie reflektiert und dies auf dem Hintergrund moralischer, rechtlicher und spiritueller Standards (Verifikation). Bei der Verifikation spielten unter anderem folgende Fragen eine Rolle:
- Wenn Du Dein eigener Klient wärst, würdest Du Dir eine Asylunterbringung zumuten?

- Wenn Du das Prinzip »Gottesebenbildlichkeit « (11) in der Sozialen Arbeit meditierst, was hätte das für Konsequenzen für Deine Praxis?

- Wenn Du Soziale Arbeit als Menschenrechtspraxis verstehst, wie müsste die Wohnungslosenhilfe dann gestaltet werden? (12)

»Umwenden« bedeutet: Während der Verifikation einen Perspektivwechsel im Sinne der reflektierten Standards zu versuchen und gegebenenfalls einen Musterwechsel (»Loslassen«) als Grundlage zukünftiger Handlungen zu probieren. Auf diese Weise wurde die ambulante Wohnungslosenhilfe entwickelt. Damals waren das marginale Angebote; heute folgen über 80 Prozent der Angebote den Prinzipien Ambulanter Hilfe. (13)

Diese Vorgehensweise wird übrigens im Kontext neuer Konzepte der Aktionsforschung als typische für eine innovative Praxis bezeichnet. (14) Das Eindrucksvolle an den neuen Konzepten der Aktionsforschung ist die Tatsache, dass sie uns in eine Nachdenklichkeit im Zusammenhang mit notwendigen Handlungsoptionen zwingt. Die Autoren analysieren die geschichtlichen Phasen des Wirtschaftens und ordnen diese verschiedene Bewusstseinsmodalitäten zu:
- prämodernes Bewusstsein
- traditionelles Bewusstsein
- egozentrisches Bewusstsein
- Stakeholder-zentrisches Bewusstsein
- ökozentrisches Bewusstsein

und entwickeln Strategien, wie wir durch eine integrierte Vorgehensweise (micro, meso, macro, mundo) aus einer globalen Misswirtschaft herauskommen können: »Der ökologische Abgrund (Überstrapazierung), der soziale Abgrund (Ungleichheit und Armut) und der spirituellkulturelle Abgrund (Depression) sind die Folge eines ökonomischen Denkmodells, das die Erde, den Menschen und Geld als Ware versteht. Doch die Erde ist keine Ware und das Gleiche gilt für den Menschen. Wir haben diese Erde nicht geschaffen; sie wurde uns geschenkt.« (a. a. O., S. 165)

Offensichtlich ist es so, dass wir uns mit unserer europäischen Glaubensgeschich- 
te wieder auseinandersetzen müssen und nachdem der Kapitalismus die »äußerlichen Heiligen « abgeschafft hat, müssen wir uns wohl auf den Weg machen »in ihm selbst aber das Bedürfnis nach dem Absoluten wiederzuerwecken «. (15) Dies wäre eine Bewusstseinsweise, die uns vor Daseinsgefräßigkeit und Machbarkeitswahn schützen könnte und uns auch den Weg zu einem ökozentrierten Management ebnen könnte.

\section{Anmerkungen}

(1) Vergl. dazu beispielsweise das Interview mit dem Autor Yural Noah Harari (»Homo Deus - Eine Geschichte von Morgen «, München 2017) in »Der Spiegel « 12 (2017), S. 104-107.

(2) Arnold Gehlen, Die Seele im technischen Zeitalter. Sozialpsychologische Probleme in der industriellen Gesellschaft, Hamburg 1957.

(3) Vergl. dazu die Johannesoffenbarung Kap. 17+18 in der Übersetzung der "Bibel in gerechter Sprache", Gütersloh 2007 (3. Auflage), S. 2.272-2.274.
(4) Ulrike Sals, Die »Hure Babylon«, in: Wörterbuch alttestamentlicher Motive, Darmstadt 2013, S. 242.

(5) In: Friedrich H. Tenbruck, Die kulturellen Grundlagen der Gesellschaft. Der Fall der Moderne, Opladen 1989, S. 126-142.

(6) Vergl. dazu: Humanistischer Pressedienst vom 1.2.2017.

(7) Vergl. dazu: Anton A. Bucher, Psychologie der Spiritualität. Handbuch, Weinheim, Basel 2007, S. 42 f.

(8) Diese Zusammenhänge können nur angedeutet werden. Neben dem zitierten Hinweis von Gregor Gysi (vergl. Fußnote 6) wäre beispielsweise auf einen Aufsatz des Sozialwissenschaftlers, Journalisten und Publizisten Erhard O. Müller (Gründungsmitglied der Partei »Die Grünen«) zu verweisen: Gott und die Linke. Politik muss sich den religiösen Grundbedürfnissen der Menschen stellen. Über Spiritualität und gesellschaftliches Handeln, in: »Frankfurter Rundschau « vom 24.12.2001

(9) Erich Fromm, Ihr werdet sein wie Gott, Reinbek bei Hamburg 1980, S. 49-53.
(10) Meister Eckhart, Mystische Schriften, Frankfurt am Main 1991, S. 173.

(11) Dieses Bild schließt an ein Märchen der Brüder Grimm an, das in ihrer Sammlung »Kinder- und Hausmärchen « die Nr. 87 trägt: »Der Arme und der Reiche«. Es berichtet, dass der liebe Gott, als er noch unter den Menschen wandelte, obdachlos geworden sei und eine Unterkunft gesucht habe.

(12) Vergl. dazu: Bernd Rothenberger, Moralpsychologie und sozialethische Probleme. Grundlagen und Strategien der Durchsetzung von Gerechtigkeit und Menschenrechten, München 1985.

(13) Vergl. dazu: Andreas Strunk, Ambulante Wohnungslosenhilfe, Stuttgart 2019.

(14) Vergl. dazu: C. Otto Scharmer, Katrin Käufer, Von der Zukunft her führen. Theorie $U$ in der Praxis. Heidelberg 2014.

(15) Maurice Merleau - Ponty, Die Abenteuer der Dialektik, Frankfurt am Main 1968, S. 30.

\section{Digitalisierung - da kann ich mitmachen!}

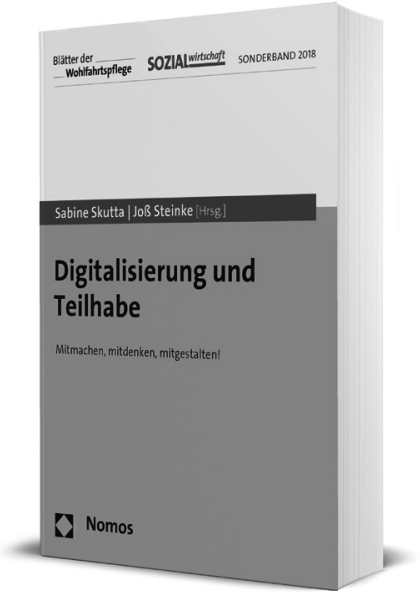

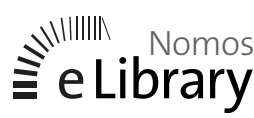

\author{
Digitalisierung und Teilhabe \\ Mitmachen, mitdenken, mitgestalten! \\ Sonderband 2018 der Zeitschriften \\ Blätter der Wohlfahrtspflege und Sozialwirtschaft \\ Herausgegeben von Dr. Sabine Skutta und Dr. Joß Steinke, et al. \\ 2019, 315 S., brosch., 59,- €, (für Bezieher der Zeitschrift \\ im Rahmen des Abonnements 44,- $€$ ) \\ ISBN 978-3-8487-5250-8 \\ nomos-shop.de/39680
}

Digitalisierung verspricht ein Mehr an Partizipation - zumindest als Möglichkeit. Was braucht es, um diese Chancen auch wahrzunehmen? Expertinnen und Experten zu politischen, ethischen und technischen Fragen sowie zur Umsetzung in der Praxis sozialer Arbeit gehen dieser Frage nach.
Bestellen Sie im Buchhandel oder versandkostenfrei online unter nomos-shop.de Bestell-Hotline (+49)7221.2104-37 | E-Mail bestellung@nomos.de | Fax (+49)7221.2104-43 Alle Preise inkl. Mehrwertsteuer
Nomos 